\title{
Influence of treatments on the dielectric properties of sugar palm fiber reinforced phenolic composites
}

\begin{abstract}
The dielectric properties of sugar palm fiber (SPF) reinforced-phenolic (PF) composites have been studied in terms of bonding between fiber and matrix. The paper aims to investigate the effect of alkaline treatment and sea water treatment on SPF composite using the dielectric relaxation spectroscopy in the frequency range from $0.1 \mathrm{~Hz}$ to $0.1 \mathrm{MHz}$ and temperature range from $80{ }^{\circ} \mathrm{C}$ to $200^{\circ} \mathrm{C}$. The results were discussed in terms of dynamic molecular and interfacial process. Our analysis suggests that interfacial adhesion in the case of alkaline treated composite is higher than those of untreated and sea water treated composites.
\end{abstract}

Keyword: Sugar palm fiber; Dielectric properties; Alkaline treatment; Sea water treatment 